ARTICLE

\title{
Momentum-resolved visualization of electronic evolution in doping a Mott insulator
}

Cheng Hu (1) 1,2,6,7, Jianfa Zhao 1,2,7, Qiang Gao1,2, Hongtao Yan¹,2, Hongtao Rong1,2, Jianwei Huang ${ }^{1,2}$, Jing Liu 1,2,3,

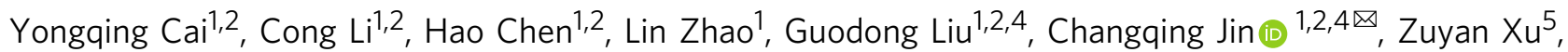
Tao Xiang ${ }^{1,2,3,4} \&$ X. J. Zhou (iD ${ }^{1,2,3,4 凶}$

High temperature superconductivity in cuprates arises from doping a parent Mott insulator by electrons or holes. A central issue is how the Mott gap evolves and the low-energy states emerge with doping. Here we report angle-resolved photoemission spectroscopy measurements on a cuprate parent compound by sequential in situ electron doping. The chemical potential jumps to the bottom of the upper Hubbard band upon a slight electron doping, making it possible to directly visualize the charge transfer band and the full Mott gap region. With increasing doping, the Mott gap rapidly collapses due to the spectral weight transfer from the charge transfer band to the gapped region and the induced low-energy states emerge in a wide energy range inside the Mott gap. These results provide key information on the electronic evolution in doping a Mott insulator and establish a basis for developing microscopic theories for cuprate superconductivity.

\footnotetext{
${ }^{1}$ National Lab for Superconductivity, Beijing National Laboratory for Condensed Matter Physics, Institute of Physics, Chinese Academy of Sciences, Beijing, China. ${ }^{2}$ University of Chinese Academy of Sciences, Beijing, China. ${ }^{3}$ Beijing Academy of Quantum Information Sciences, Beijing, China. ${ }^{4}$ Songshan Lake Materials Laboratory, Dongguan, China. ${ }^{5}$ Technical Institute of Physics and Chemistry, Chinese Academy of Sciences, Beijing, China. ${ }^{6}$ Present address: Department of Materials Science and Engineering, Cornell University, Ithaca, NY, USA. ${ }^{7}$ These authors contributed equally: Cheng Hu, Jianfa Zhao.

凶email: Jin@iphy.ac.cn; XJZhou@iphy.ac.cn
} 
n high-temperature cuprate superconductors, the $\mathrm{CuO}_{2}$ plane is a common and key structural element that dictates their electronic structure and unusual physical properties. In the undoped parent compound, each $\mathrm{Cu}$ site in the $\mathrm{CuO}_{2}$ plane has nine electrons in the $\mathrm{Cu} 3 d$ orbitals, which is expected to give a half-filled band and a metallic state according to the traditional band theory ${ }^{1,2}$. Instead, it becomes an insulator because of the strong electron-electron correlation that splits the initial halffilled band into the lower Hubbard band (LHB) and upper Hubbard band (UHB) separated by the on-site Coulomb repulsion $\mathrm{U}$ (Fig. 1a) ${ }^{3}$. The hybridization of the lower $\mathrm{Cu} 3 d$ states and $\mathrm{O} 2 p$ states gives rise to a charge-transfer band (CTB) in between the lower and upper Hubbard bands, resulting in a correlated insulator with a charge transfer gap $\Delta$ (Fig. 1a) ${ }^{3}$. The cuprate parent compound is, therefore, strictly speaking, a charge-transfer insulator ${ }^{3}$ although it is generally believed that it can be described by an effective Hubbard Hamiltonian where the charge-transfer band substitutes for the lower Hubbard band and the chargetransfer gap $\Delta$ plays the role of $U$ (Fig. 1a) ${ }^{4,5}$.

High-temperature superconductivity in cuprates is realized by doping the $\mathrm{CuO}_{2}$ planes with charge carriers (holes or electrons $)^{4,5}$. A key question is how the insulating state of the parent compound is transformed into a conductive or superconducting state upon carrier doping. Such information on the electronic structure of the parent compound and its doping evolution is essential for establishing theories and understanding the origin of high-temperature superconductivity ${ }^{6}$. Specifically, prominent issues are related to how the Mott gap evolves and how the low-lying electronic states emerge with doping $^{7-10}$. The tunneling experiment, owing to its advantage in measuring both the occupied state and unoccupied state, has provided some key insight on the Mott gap in the parent compound $^{11,12}$ and the emergence of low-lying states inside the gap upon slight carrier doping ${ }^{13-15}$. However, because the tunneling experiment measures the local density of states, it lacks the momentum information of electrons that is required in describing the complete electronic structure ${ }^{1-15}$. Angleresolved photoemission spectroscopy (ARPES), with its unique momentum-resolving power, has played a key role in studying the electronic structure of high-temperature cuprate superconductors $^{16}$. However, because ARPES can only measure the occupied state, and the chemical potential of the parent compounds and hole-doped compounds lies inside the Mott gap (Fig. 1a), the ARPES measurements have not been possible to probe the entire gapped region in studying the parent compounds ${ }^{17-21}$ and the evolution of the Mott gap and the lowlying states in lightly hole-doped cuprates ${ }^{22-25}$. Little information has been available on the momentum-resolved electronic structure evolution of the lightly doped cuprates that can cover the full energy spectrum of the gapped region ${ }^{26}$.

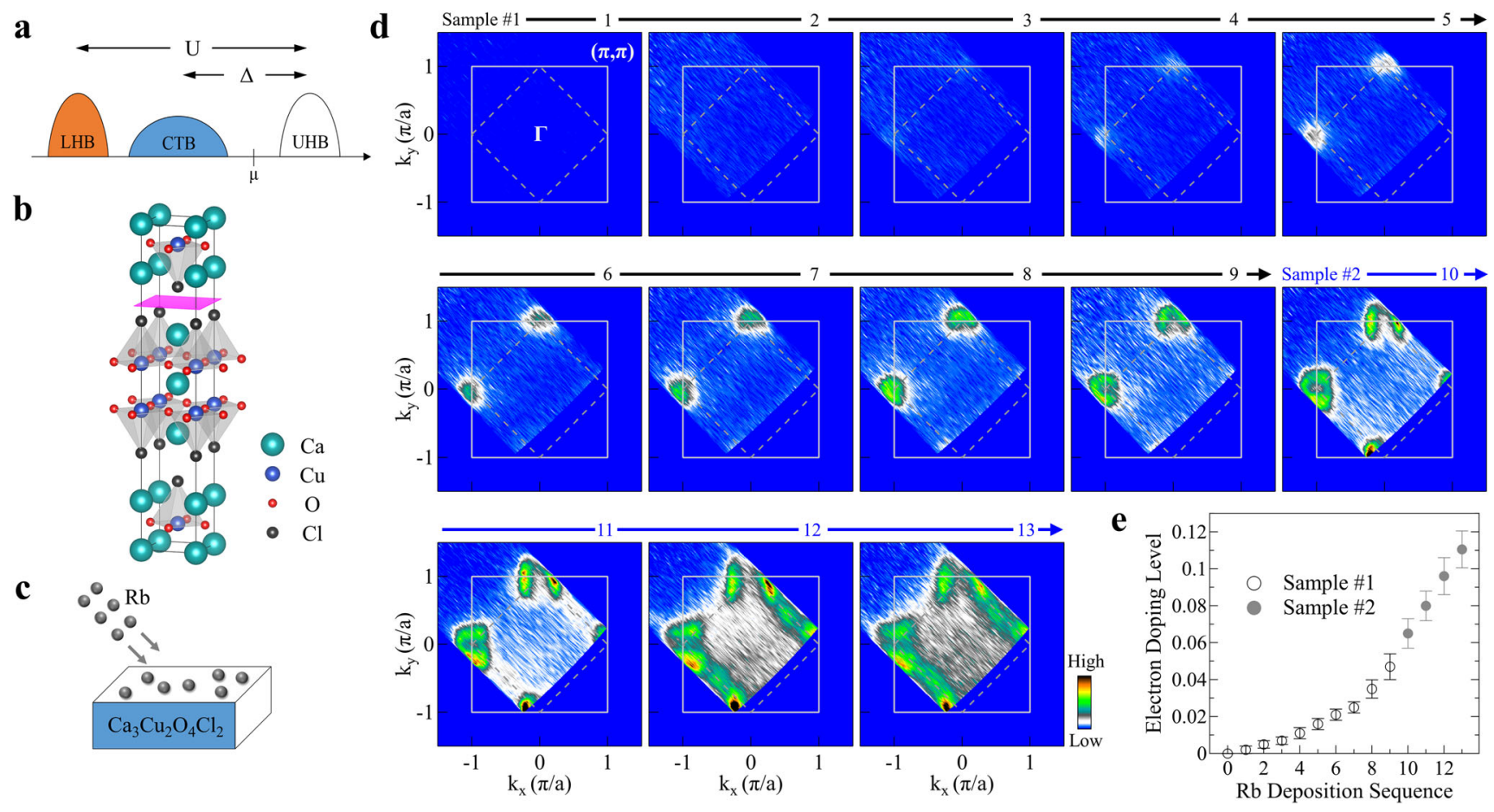

Fig. 1 Fermi surface evolution of $\mathbf{C a}_{\mathbf{3}} \mathbf{C u}_{\mathbf{2}} \mathbf{O}_{\mathbf{4}} \mathbf{C l}_{\mathbf{2}}$ with $\mathbf{R b}$ deposition. a Schematic electronic structure picture of the cuprate parent compound. It consists of

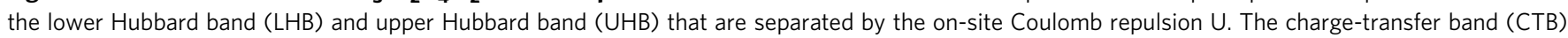

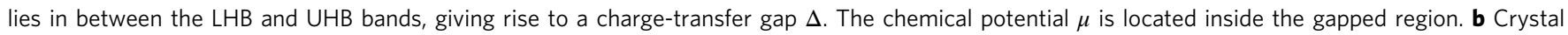
structure of $\mathrm{CCOC} 326$ consisting of double $\mathrm{CuO}_{2}$ planes in one structural unit. The crystal cleaves between two adjacent $\mathrm{CaCl}$ layers, as marked by the pink sheet. c Schematic illustration of in situ Rb deposition onto the sample surface. Electrons are transferred into the top layers due to the physical

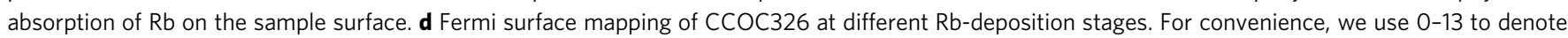
the deposition sequence where 0 represents the original sample before $\mathrm{Rb}$ deposition. The images for sequences $0-9$ are obtained on one sample while sequences 10-13 are obtained from another sample that complements each other. The Fermi surface images are obtained by integrating the spectral weight over an energy window of $[-0.03,0.03] \mathrm{eV}$ with respect to the Fermi level. e Estimated electron doping level after each Rb-deposition stage. For the deposition stages from 3 to 11, the electron doping level is calculated from the area of the electron-like Fermi surface centered at ( $-\pi$, 0 ). For the deposition sequences 12 and 13, it is estimated from the area of the hole-like Fermi surface centered at $(-\pi, \pi)$. The doping level for deposition stages 1 and 2 are obtained by linear extrapolation in the low doping region according to the deposition procedures. Error bars reflect the uncertainty in determining the Fermi momentum. 
Recently, in situ alkali metal deposition provides an effective way to fine-tune surface electron doping in correlated systems, such as cuprates $^{27-29}$, iridates ${ }^{30-32}$, and iron-based superconductors ${ }^{33-35}$. Yet, to our knowledge, there has been no report of ARPES experiment with alkali metal deposition on cuprate parent compounds. It is challenging for ARPES experiments on these insulators because the measurement temperature usually needs to be high, say $\sim 100 \mathrm{~K}$ for $\mathrm{Ca}_{2} \mathrm{CuO}_{2} \mathrm{Cl}_{2}{ }^{18}$, to avoid sample charging which hinders sufficient amount of the deposited alkali metal from staying stable on the sample surface.

In this paper, we report our ARPES study on the electronic structure evolution in doping $\mathrm{Ca}_{3} \mathrm{Cu}_{2} \mathrm{O}_{4} \mathrm{Cl}_{2}$ (CCOC326), a double-layer parent compound of high-temperature cuprate superconductors. Fine-tuning of the electron doping is realized by in situ depositing $\mathrm{Rb}$ onto the sample surface. As soon as the CCOC326 parent compound is slightly electron-doped, we find that the chemical potential jumps from the position inside the Mott gap to the bottom edge of the upper Hubbard band. This makes it possible for ARPES to see the full Mott gap, study its doping evolution and keep track of the doping-induced emergence of the low-lying electronic states. The observed Mott gap represents an indirect gap of $\sim 1.5 \mathrm{eV}$ between the charge-transfer band top at $(\pi / 2, \pi / 2)$ and the upper Hubbard band bottom at $(\pi$, $0)$. With electron doping, the Mott gap shows little change in its magnitude but it rapidly collapses due to the spectral weight depletion of the charge-transfer band and the emergence of lowlying states in the gapped region. Distinct doping evolution of the low-energy states is observed between the $(\pi / 2, \pi / 2)$ nodal region and the $(\pi, 0)$ Brillouin zone face. Upon a slight electron doping, the upper Hubbard band is filled with electrons and a metallic electron-like band develops around the $(\pi, 0)$ zone face. In the meantime, near the $(\pi / 2, \pi / 2)$ nodal region, broad in-gap states emerge inside the gapped region spreading over a wide energy range and approach the chemical potential with increasing doping. These results provide momentum-resolved information on the electronic structure evolution in lightly doping a Mott insulator over the entire gapped region and establish a basis for developing microscopic theories in understanding unusual physical properties and high-temperature superconductivity in cuprate superconductors.

\section{Results}

Evolution of the Fermi surface. The $\mathrm{Ca}_{3} \mathrm{Cu}_{2} \mathrm{O}_{4} \mathrm{Cl}_{2}$ represents a prototypical parent compound of high-temperature cuprate superconductors ${ }^{36}$. Its crystal structure consists of two $\mathrm{CuO}_{2}$ planes in one structural unit (Fig. 1b) and is analogous to $\mathrm{Ca}_{2} \mathrm{CuO}_{2} \mathrm{Cl}_{2}$ (CCOC214) with one $\mathrm{CuO}_{2}$ plane in a unit cell ${ }^{18,21}$. We choose to work on this CCOC326 parent compound because it is less insulating than its single-layer counterpart CCOC214 ${ }^{37}$, so that our ARPES measurements can be performed at a lower temperature without sample charging problems (see Supplementary Fig. 1). The electronic structure of CCOC326 (Supplementary Fig. 2) is similar to that of CCOC $214^{18,21}$ but with a smaller energy gap and sharper spectral features. We deposit Rb onto the sample surface to fine-tune the electron doping level (Fig. 1c); this can realize systematic ARPES measurements on the same sample under the same experimental condition. The lower measurement temperature of CCOC 326 is crucial for the success of our present work because only at low temperature can a sufficient amount of $\mathrm{Rb}$ be absorbed on the sample surface and stay stable to provide high enough electron doping.

Figure $1 \mathrm{~d}$ shows the Fermi surface mapping of $\mathrm{Ca}_{3} \mathrm{Cu}_{2} \mathrm{O}_{4} \mathrm{Cl}_{2}$ at different $\mathrm{Rb}$-deposition stages. Little spectral weight is observed for the initial two stages ( 1 and 2), which is consistent with the insulating nature of the CCOC326 parent compound (see
Supplementary Fig. 2). The spectral weight starts to appear after stage 3 near the $(0, \pi)$ and $(-\pi, 0)$ regions in the covered momentum space. It gets stronger with further $\mathrm{Rb}$ deposition till stage 8 . Then an electron-like Fermi pocket is observed for stage 9 and its size increases with Rb deposition. For stage 11, the spectral weight near the nodal region starts to emerge and gets stronger with further $\mathrm{Rb}$ deposition. The overall Fermi surface evolution with $\mathrm{Rb}$ deposition is similar to the previous ARPES measurements on typical electron-doped cuprates $(\mathrm{Nd}, \mathrm{Ce})_{2} \mathrm{CuO}_{4}{ }^{26}$, confirming that electron doping is realized in the CCOC326 parent compound by $\mathrm{Rb}$ deposition. By the Luttinger sum rule 26,38 , the electron doping level $\mathrm{p}$ is calculated from the observed Fermi surface area for the later deposition stages, and estimated for the initial stages (1 and 2) from the linear extrapolation according to the deposition procedures, as shown in Fig. 1e. We see that the doping level is well controlled under the sequential $\mathrm{Rb}$ deposition, in particular, it covers the lightly doped region ( $p \sim 0-0.04)$ which is the key range to keep track of the electronic structure evolution from the parent compound. This regime is the main focus of this work that was not covered in the previous ARPES measurements ${ }^{5,26,38}$. Considering that CCOC326 can also be doped with holes to reach superconductivity ${ }^{39,40}$, our present work indicates that this double-layer cuprate parent compound $\mathrm{Ca}_{3} \mathrm{Cu}_{2} \mathrm{O}_{4} \mathrm{Cl}_{2}$ is a new ambipolar cuprate system that can be doped by either holes or electrons, in addition to limited cases found in other cuprate superconductors ${ }^{15,41}$.

Evolution of the band structure. Figure 2 shows the band structure evolution with $\mathrm{Rb}$ deposition measured along with three high symmetry momentum cuts, $(-\pi,-\pi)-(\pi, \pi)$ nodal direction (Fig. $2 \mathrm{a}),(-\pi, 0)-(0, \pi)$ cut crossing both the $(-\pi / 2,-\pi / 2)$ nodal point and $(0, \pi)$ antinodal point (Fig. $2 \mathrm{~b})$, and $(-\pi,-\pi)-(-\pi, \pi)$ cut along the zone face (Fig. 2c). For the undoped CCOC326, its band structure is characterized by the charge-transfer band that shows a clear dispersion along the nodal direction with its top $\sim 300 \mathrm{meV}$ below the Fermi level (leftmost panel in Fig. 2a) and a nearly vertical band along the zone face with its top $\sim 600 \mathrm{meV}$ below the Fermi level (leftmost panel in Fig. 2c) (see also Supplementary Fig. 2). As soon as the sample is doped with a tiny amount of electrons $(<0.005$ for stages 1 and 2 in Fig. 2), both bands (second and third panels from left in Fig. 2a, c) abruptly sink down by $\sim 1.1 \mathrm{eV}$. As will be discussed later, this is due to the chemical potential jump to the bottom edge of the upper Hubbard band. This makes it possible for ARPES to directly access the entire Mott gap in the cuprate parent compound, which is an indirect gap defined by the top of the charge-transfer band at the $(\pi / 2, \pi / 2)$ nodal point and the bottom of the upper Hubbard band at the $(\pi, 0)$ antinodal point. The gap size thus determined is $\sim 1.5 \mathrm{eV}$. This agrees with the gap value measured directly by the tunneling experiments ${ }^{12}$. These results indicate that, right after a slight electron doping, the chemical potential shifts to the bottom edge of the upper Hubbard band. With further electron doping, more states are created in the upper Hubbard band, gradually forming a parabolic electron band near $(\pi, 0)$. In this case, the chemical potential is defined by this newly formed band in the upper Hubbard band. This is consistent with the usual picture that is proposed in literature ${ }^{5,26}$.

From Fig. 2, we can directly visualize how the low-lying electronic states emerge and evolve inside the Mott gap with increasing electron doping. Overall, with the introduction of electrons, new states appear inside the gap; their spectral weight gets stronger with increasing electron doping. Also, the newly developed states spread over a wide energy range inside the gap region, indicating the incoherent nature of the correlated 


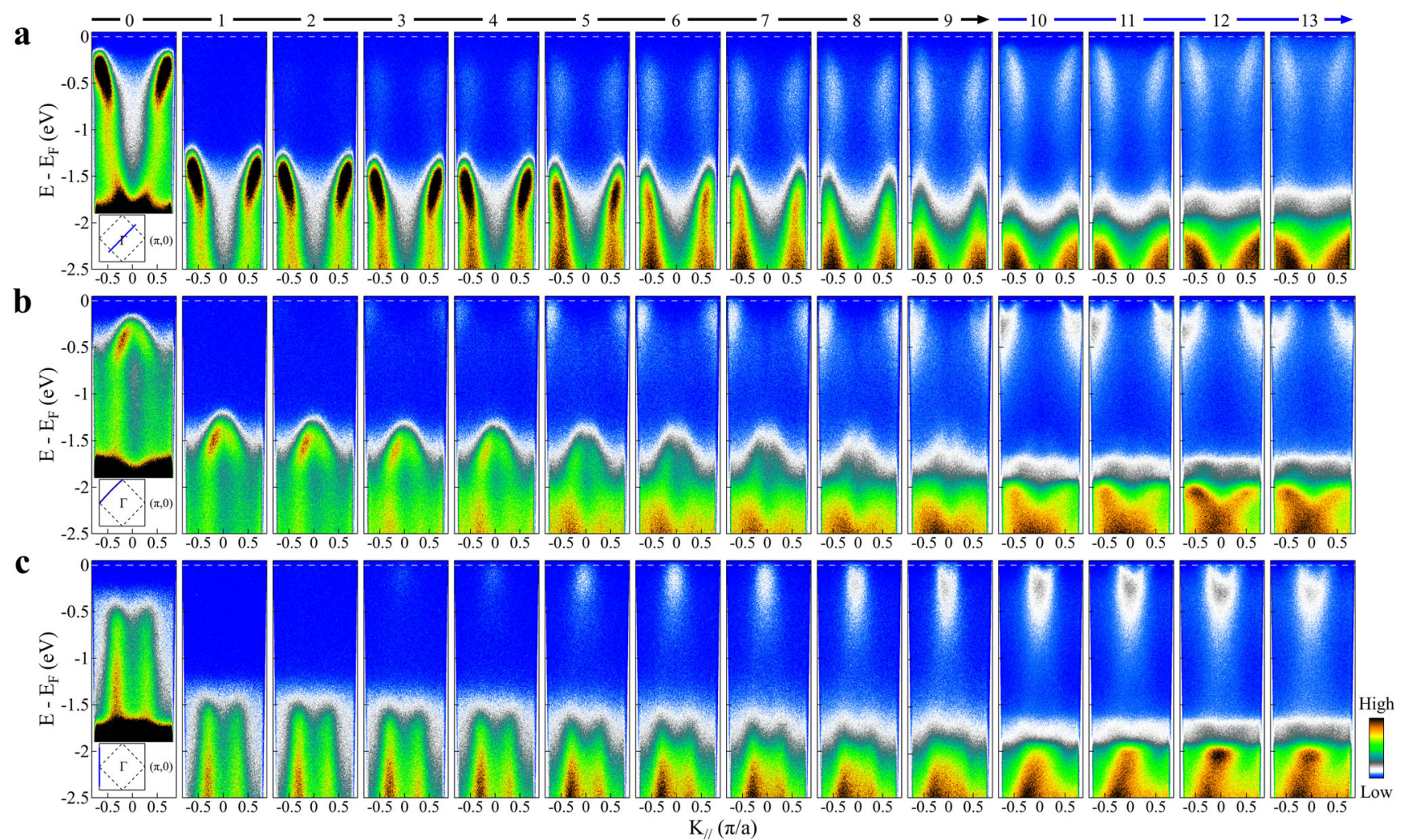

Fig. 2 Band structure evolution of $\mathbf{C a}_{\mathbf{3}} \mathbf{C u}_{\mathbf{2}} \mathbf{O}_{\mathbf{4}} \mathbf{C l}$ with $\mathbf{R} \mathbf{b}$ deposition. a Doping evolution of band structure along with the $(-\pi / 2,-\pi / 2)-(\pi / 2, \pi / 2)$ nodal momentum cut. $\mathbf{b}$ Band structure evolution with $\mathrm{Rb}$ deposition along the $(-\pi, 0)-(0, \pi)$ momentum cut. c Band structure evolution with $\mathrm{Rb}$ deposition along with the $(-\pi,-\pi)-(-\pi, \pi)$ cut. The locations of the three momentum cuts are illustrated by the blue solid line in the inset of panels (a), (b), and (c), respectively.

electronic states. In the meantime, the spectral weight of the charge-transfer band decreases with the doping increase. In particular, the low-lying in-gap states exhibit distinct momentum-dependence; their behavior along the nodal direction (Fig. 2a) is markedly different from that along the zone face (Fig. 2c). Along the zone face (Fig. 2c), the low-energy states appear starting from the Fermi level, then spread downward to high-binding energy. With increasing doping, their spectral weight gets stronger. At high doping (stages 9-13 in Fig. 2c), coherent parabolic electron-like bands form near the $(\pi, 0)$ region. Note that, along the zone face, the newly developed in-gap states lie at the Fermi level as soon as they appear upon tiny electron doping. On the other hand, along the nodal direction (Fig. 2a), the in-gap states appear from the high-binding energy near the top of the charge-transfer band, spreading upwards with the increase of the spectral weight with increasing electron doping. However, the bands do not cross the Fermi level at lower doping but gradually approach the Fermi level with increasing doping. The band reaches the Fermi level only at high doping (stages 12 and 13 in Fig. 2a). We find that the newly developed ingap states along the nodal direction (Fig. 2a) have quite similar "waterfall" band dispersion as the original charge-transfer band. This is different for the in-gap states along the zone face where they exhibit a quite different band dispersion as the original vertical bands. It is also interesting to notice that, the band evolution with electron doping along the nodal direction in the present work is reminiscent of that observed in lightly hole-doped cuprates $^{22-25}$ although the behavior near the $(\pi, 0)$ antinodal region is completely different between the lightly electron-doped and lightly hole-doped cases. In addition to the evolution of lowlying electronic states mentioned above, we also observe a profound spectral change in the high-binding energy region from
2 to $2.5 \mathrm{eV}$ with increasing electron doping. The observation of such a strong modification is important because generally it is not expected to see such a strong change in such a high-energy region with electron doping.

We also note that, when the deposited alkali metal is thick enough to form full layers, they may produce free-electron-like bands that are centered around the Brillouin zone center $(\Gamma \text { point })^{31}$. In our measurements, we do not observe any free-electron-like bands near the Brillouin zone center. Moreover, the observed in-gap states lie near the $(\pi / 2, \pi / 2)$ nodal region and $(\pi, 0)$ antinodal region. This can fully rule out the possibility that the in-gap states originate from the $\mathrm{Rb}$ valence band; they represent the intrinsic features of the doped cuprate parent compounds.

Collapse of the Mott gap. Figure 3 shows how the chargetransfer band and the low-lying in-gap states evolve with electron doping in terms of their energy positions and spectral weight. Figure $3 \mathrm{a}, \mathrm{b}$ shows the photoemission spectra (energy-distribution curves, EDCs) at $(\pi / 2, \pi / 2)$ nodal point and $(\pi, 0)$ antinodal point, respectively, at different Rb-deposition stages. The EDCs at $(\pi / 2, \pi / 2)$ nodal point (Fig. 3a) are characterized by two main features: one is the well-defined peak (marked by solid circles in Fig. 3a) from the charge-transfer band which loses its weight with increasing doping, and the other is the broad feature inside the gapped region between $[-1,0] \mathrm{eV}$ forming the low-lying electronic states that gain weight with increasing doping. For the EDCs at $(\pi, 0)$ antinodal point (Fig. $3 b$ ), in addition to the feature near $-1.5 \mathrm{eV}$ in the doped samples that is related to the chargetransfer band, the main feature is the in-gap states that grow up in intensity with increasing doping and form a peak marked by triangles in Fig. 3b. Figure $3 c$ shows the energy position of the 

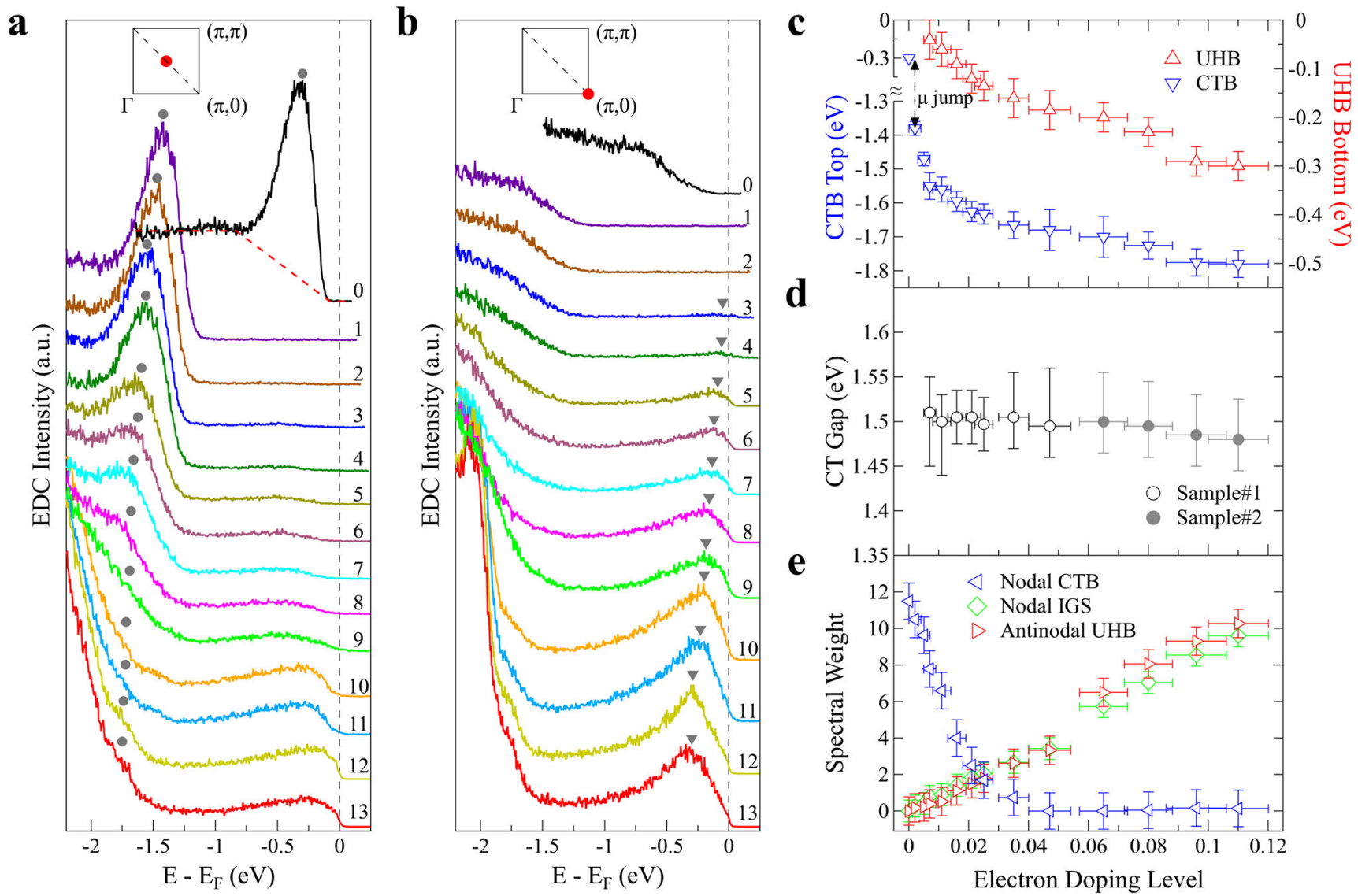

Fig. 3 Doping evolution of the charge-transfer band (CTB), upper Hubbard band (UHB), and the low-energy in-gap states (IGS). a Doping evolution of EDCs taken at $(\pi / 2, \pi / 2)$ point. The peak position of the EDCs, marked by gray circles, defines the top of the nodal charge-transfer band. $\mathbf{b}$ Doping evolution of EDCs taken at $(\pi, 0)$ point. The peak position of the EDCs, marked by gray triangles, represents the bottom of the antinodal upper Hubbard band. c The EDC peak positions of the nodal charge-transfer band and antinodal upper Hubbard band extracted from (a) and (b), respectively. $\mathbf{d}$ The magnitude of the charge-transfer gap (CT Gap) determined from the difference of the nodal CTB and antinodal UHB positions in (c). e Doping evolution of the spectral weight of the nodal CTB (blue triangles), antinodal UHB (red triangles), and nodal in-gap states (IGS) (green diamonds). The nodal CTB spectral weight is obtained from the EDC peak area in (a) after subtracting the linear background as illustrated by the red dashed line in (a) for the EDC of the undoped sample. The spectral weight of antinodal UHB and nodal IGS is obtained by integrating the EDCs in (b) and (a) over an energy window $[-1$, $0.03] \mathrm{eV}$, respectively.

EDC peaks at $(\pi / 2, \pi / 2)$ (marked by solid circles in Fig. 3a) related to the top position of the charge-transfer band, and that of the EDC peaks at $(\pi, 0)$ (marked by triangles in Fig. $3 \mathrm{~b}$ ) related to the bottom position of the upper Hubbard band. Both positions show a jump right after the electron doping, and then exhibit a gradual shift to higher binding energy with increasing electron doping (Fig. 3c). Their energy difference (Fig. 3d) represents the Mott gap magnitude that shows little change with the doping level and stays around $\sim 1.5 \mathrm{eV}$. On the other hand, the EDCs at $(\pi / 2$, $\pi / 2$ ), which is the top of the charge-transfer band, lose spectral weight rapidly with initial electron doping (Fig. 3a, e); the spectral weight of the charge-transfer band nearly disappears at a doping level of $\sim 0.04$ (Fig. 3e). The spectral weight of the in-gap states, either at $(\pi / 2, \pi / 2)$ or $(\pi, 0)$ points, keeps increasing with electron doping, as shown in Fig. 3a, b, e. These results indicate that, with electron doping, although the magnitude of the Mott gap shows little change, the gap collapses due to the vanishing of the initial charge-transfer band, and the filling-up of the in-gap states across the entire gapped region.

Chemical potential shift. One key issue in doping the parent compounds of the cuprate superconductors is how the chemical potential shifts with doping; whether it is pinned inside the gapped region to form the confined in-gap states or it shifts to the top of the charge-transfer band or the bottom of the upper Hubbard band upon respective hole- or electron doping 4,5,7-10,16. Our detailed doping evolution of the band structure (Fig. 2) clearly indicates that, upon tiny electron doping, the chemical potential $\mu$, which lies close to the charge-transfer band in the parent compound, shifts immediately to the bottom of the upper Hubbard band. Photoemission can measure the relative shift of the chemical potential as a function of carrier doping with reference to the electronic states located far away from the Fermi level thus their energies should be qualitatively unaffected by doping $^{23,42}$. To this end, we carried out photoemission measurements on high-binding energy $\mathrm{O} 2 p$ nonbonding states which show up as sharp peaks at $\Gamma$ (Fig. $4 \mathrm{a})$ and at $(\pi, \pi)$ point (Fig. $4 \mathrm{~b}$ ), and the $\mathrm{Ca} 3 p$ core level near the binding energy of $\sim 25 \mathrm{eV}$ (Fig. 4c) in CCOC326 at different Rb-deposition stages. A consistent result on the relative chemical potential shift with electron doping in CCOC326 is obtained in Fig. 4d by keeping track of the relative EDC peak position change with $\mathrm{Rb}$ deposition from the three cases in Fig. $4 \mathrm{a}-\mathrm{c}$. A chemical potential jump of $\sim 1.0 \mathrm{eV}$ is observed right after a tiny amount of electrons are introduced into the parent compound (Fig. 4d). We note that since the parent compound is a perfect insulator, the chemical potential is not well-defined and may vary its position inside the gap between different samples. The magnitude of the chemical potential jump 

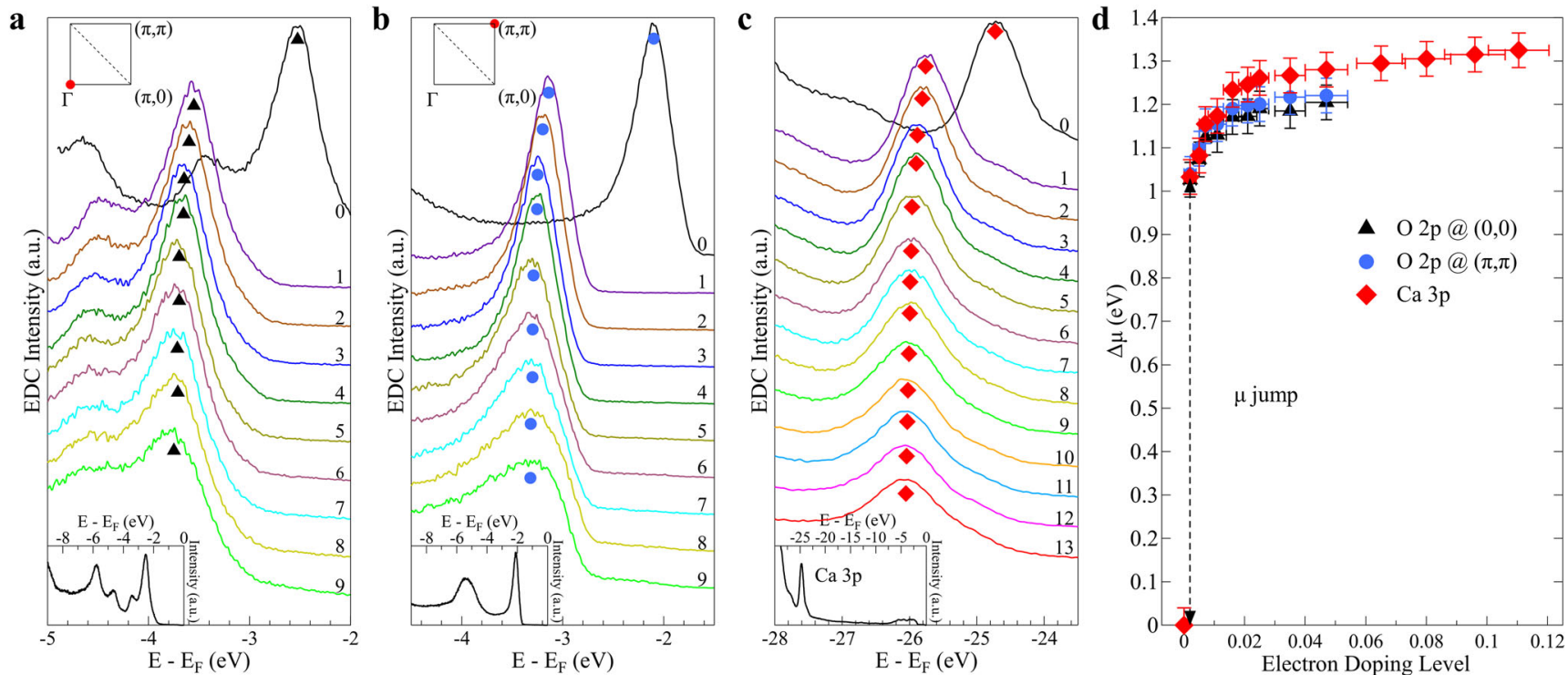

Fig. 4 Measured chemical potential shift with electron doping in $\mathbf{C a}_{3} \mathbf{C u}_{\mathbf{2}} \mathbf{O}_{\mathbf{4}} \mathbf{C l}_{\mathbf{2}}$. a-c Doping evolution of $O 2 p$ orbitals at $\Gamma$ (a) and $(\pi, \pi)(\mathbf{b})$, and $\mathrm{Ca} 3 p$ core level (c). Symbols in (a-c) indicate the respective spectral peaks. The photoemission spectra of the undoped parent compound in a larger energy scale are shown in the respective insets. $\mathbf{d}$ Chemical potential shift with electron doping determined from the three types of measurements in (a), (b), and (c). The chemical potential shift for the undoped parent compound is set at zero as a reference. The chemical potential shifts obtained from the three cases give a similar result with a jump of $\sim 1 \mathrm{eV}$ in the initial Rb-deposition stage. Error bars reflect the uncertainty in determining the EDC peak positions.

may vary slightly among different samples, but the jump itself is intrinsic because it will shift to the bottom of the upper Hubbard band as soon as the electrons are doped. As seen in Fig. 4d, upon further electron doping in CCOC326, the chemical potential gradually increases, consistent with the chemical potential shift in other electron-doped cuprates ${ }^{42}$.

It is important to investigate, after $\mathrm{Rb}$ deposition and some electrons are transferred into the $\mathrm{CuO}_{2}$ planes, whether there is a band-bending that may affect the chemical potential determination. We think the situation in Rb-deposited CCOC326 is different from the usual band-bending picture. Before $\mathrm{Rb}$ deposition, the $\mathrm{CuO}_{2}$ layer, $\mathrm{Ca}$ layer, and $\mathrm{CaCl}$ layer already have charge of $2-, 2+$, and $1+$, respectively, that form an internal electrical field in CCOC326. The charge transfer of $2 \delta$ electrons from the $\mathrm{Rb}$-deposition layer to the two $\mathrm{CuO}_{2}$ planes only slightly modifies the charge distribution. As seen in our experiments, dramatic changes in the chemical potential shift and band structure occur before $\delta \sim 0.04$. The corresponding charge change of $\mathrm{CuO}_{2}$ plane from $2-$ to $(2+0.04)-$ is small, only accounting for $2 \%$ change. It is expected that in this case, the chemical potential shift is mainly caused by the electron doping of the $\mathrm{CuO}_{2}$ plane, but not by the band-bending near the surface. In fact, there is no band-bending between the $\mathrm{Rb}$-deposition layer and the adjacent $\mathrm{CaCl}$ layer because they both have a positive charge. This is consistent with the fact the chemical potential shift obtained from $\mathrm{Ca} 3 p$ in the $\mathrm{CaCl}$ layer is consistent with that obtained from $\mathrm{O} 2 p$ in the $\mathrm{CuO}_{2}$ plane although they have different internal electric field environments. The chemical potential shift we observed is also consistent with that observed in other electron-doped cuprates in bulk form ${ }^{42}$.

In ARPES study of $\mathrm{YBa}_{2} \mathrm{Cu}_{3} \mathrm{O}_{6.5}$, in situ potassium deposition has been successfully applied to continuously tune the surface doping from heavily overdoped to underdoped regime ${ }^{27}$ and further into heavily underdoped regime ${ }^{28}$. CCOC326 is similar to $\mathrm{YBa}_{2} \mathrm{Cu}_{3} \mathrm{O}_{6.5}$ in that they both have two $\mathrm{CuO}_{2}$ planes in one structural unit. But the charge-transfer distance in CCOC326 is shorter than that in $\mathrm{YBa}_{2} \mathrm{Cu}_{3} \mathrm{O}_{6.5}$ because the former crosses one $\mathrm{CaCl}$ layer while the latter needs to cross two $\mathrm{CuO}_{x}$ and $\mathrm{BaO}$ layers. Since ARPES is a surface-sensitive technique, at $21.2 \mathrm{eV}$ photon energy, the probe depth is on the order of $\sim 10 \AA$ that matches the surface-doped region so the doping-induced change of electronic structure near the surface can be fully detected by ARPES. The ARPES measurements of K-deposited $\mathrm{YBa}_{2} \mathrm{Cu}_{3} \mathrm{O}_{6.5}$ system ${ }^{27,28}$ and $\mathrm{Bi}_{2} \mathrm{Sr}_{2} \mathrm{CaCu}_{2} \mathrm{O}_{8+\delta}{ }^{29}$ have provided direct evidence on measuring genuine doping evolution of bulk cuprates. The consistency of our results at higher doping with the ARPES measurements on bulk samples ${ }^{26}$ also indicates that our results represent genuine doping evolution of bulk cuprates.

The full picture of momentum-resolved electronic evolution. The doping-induced band structure evolution (Figs. 2 and 3), combined with the shift of the chemical potential (Fig. 4), provides a momentum-resolved electronic structure picture in electron doping the cuprate parent compound. Figure $5 \mathrm{a}, \mathrm{b}$ shows the band structure measured along $(-\pi,-\pi)-(\pi, \pi)$ nodal direction and $(-\pi,-\pi)-(-\pi, \pi)$ zone face, respectively, at several representative doping levels. Here, the relative energy scale is used and all data are plotted with reference to the chemical potential $\mu_{0}$ using the values from Fig. $4 \mathrm{~d}$, thus highlighting the change of the chemical potential with doping. In this case, it is also straightforward to visualize the electronic structure variation with doping in three energy regions: charge-transfer band (CTB) region, ingap states (IGS) inside the energy gap region, and the upper Hubbard band (UHB) region, as marked on the top of Fig. 5a. Figure $5 \mathrm{c}$, d shows the momentum-integrated EDCs obtained from Fig. 5a, b, respectively. By summing up all the spectral weight over the covered momentum space, the integrated EDC represents the local density of states along with the particular momentum cut. With electron doping, the spectral weight transfer from the charge-transfer band to the in-gap states is clear, with the spectral weight depletion of the charge-transfer band and the increase of the in-gap states.

Combining the results in Fig. $5 \mathrm{a}-\mathrm{d}$, one gets a schematic electronic structure evolution picture in a momentum-resolved manner, as shown in Fig. $5 \mathrm{e}, \mathrm{f}$ for the $(-\pi,-\pi)-(\pi, \pi)$ nodal cut and $(-\pi,-\pi)-(-\pi, \pi)$ cut, respectively. A few key points emerge in electron doping of the parent Mott insulator. First, the 

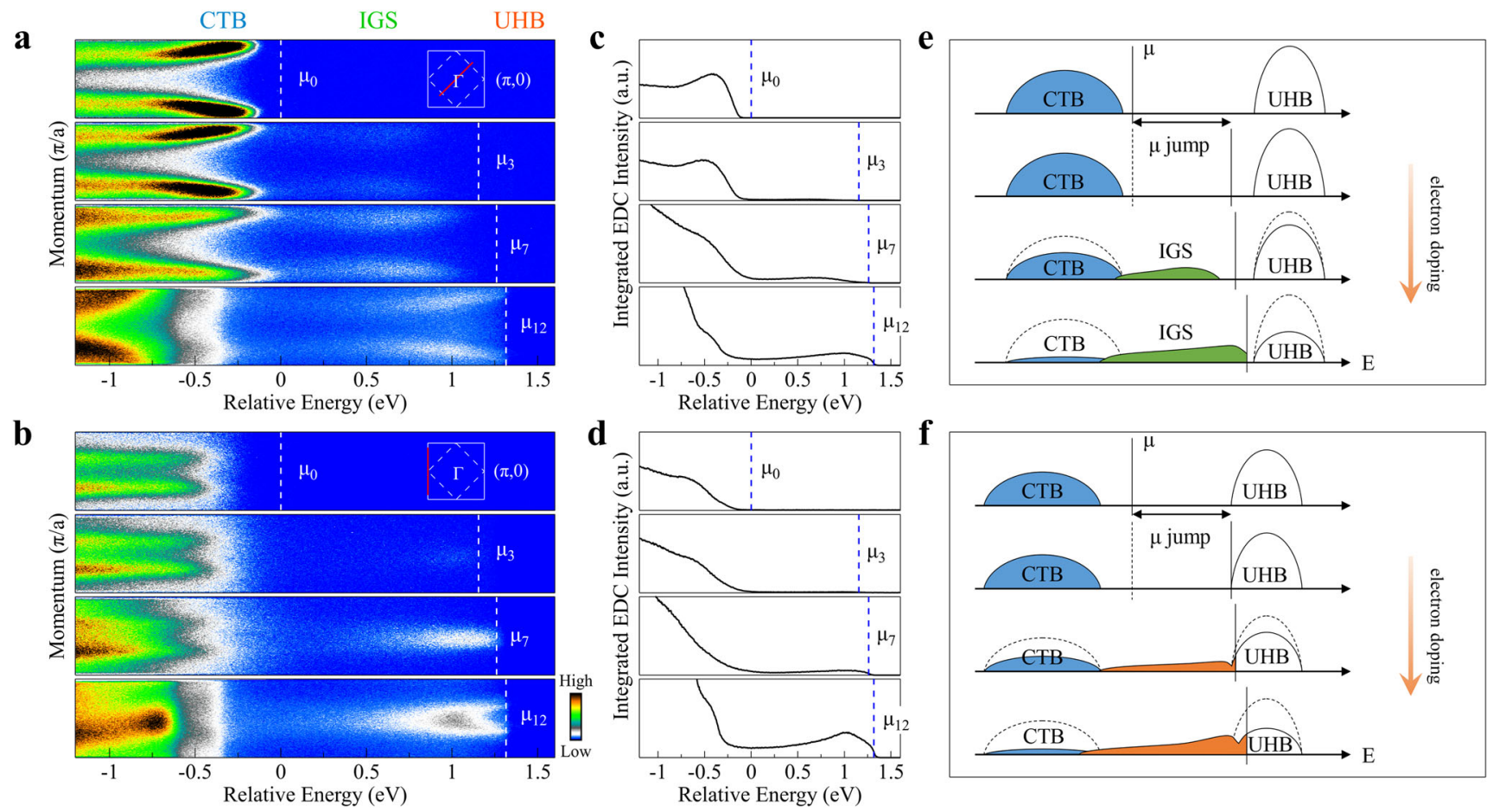

Fig. 5 Momentum-resolved electronic structure evolution picture of $\mathbf{C a}_{\mathbf{3}} \mathbf{C u}_{\mathbf{2}} \mathbf{O}_{\mathbf{4}} \mathbf{C l}_{\mathbf{2}}$ with electron doping. a Representative band structures at different electron doping levels, corresponding to Rb-deposition stages of $0,3,7$, and 12 , measured along the $(-\pi / 2,-\pi / 2)-(\pi / 2, \pi / 2)$ nodal cut. $\mathbf{b}$ Corresponding band structures at different electron doping levels measured along the $(-\pi,-\pi)-(-\pi, \pi)$ cut. The horizontal axes in (a) and (b) are plotted on a relative energy scale where the chemical potential position of the original undoped CCOC326 is set at zero, and the energy position of others is referenced to the chemical potential shift $\mu$ shown in Fig. 4d. The chemical potential positions for each doping are marked by white dashed lines. The corresponding momentum cuts for the bands are shown in the upper-right insets. c, $\mathbf{d}$ Doping evolution of the momentum-integrated EDCs which are obtained from (a) and (b), respectively, by integrating the spectral weight over the entire momentum range of the cuts shown as red lines in the insets of (a, b). e, f Schematic electronic structure evolution with electron doping along the $(-\pi / 2,-\pi / 2)-(\pi / 2, \pi / 2)$ nodal direction, and along the $(-\pi,-\pi)-(-\pi, \pi)$ zone face, respectively. The three spectral components, charge-transfer band (CTB), in-gap states (IGS), and upper Hubbard band (UHB), are also labeled on top of (a) for comparison.

chemical potential jumps from the in-gap position to the bottom of the upper Hubbard band right after a tiny amount of electron doping; second, the emergence of low-lying electronic states which span a wide energy range inside the gapped region; third, the Mott gap magnitude changes little with doping but the gap collapses due to the depletion of the charge-transfer band and the filling of the low-lying electronic states in the gapped region. Here, we note that, due to the Fermi cutoff, we have no access to the entire upper Hubbard band although one would expect that the upper Hubbard band also loses its weight with increasing electron doping ${ }^{11,43}$. Fourth, momentum anisotropy of the lowenergy in-gap states. A metallic state forms near the $(\pi, 0)$ antinodal region for the lightly doped sample, but the state near the $(\pi / 2, \pi / 2)$ nodal region is away from the Fermi level when it is formed. It gradually approaches the Fermi level with increasing doping and only crosses the Fermi level at a higher doping level above $p \sim 0.1$.

\section{Discussion}

The electronic structure of the parent compound and the lightly doped cuprates is of fundamental importance to understanding the high-temperature superconductivity mechanism and has attracted enormous theoretical attention ${ }^{4-6,44-46}$. The basic theoretical model for describing the half-filled cuprates, with a single electron on the $\mathrm{Cu} d_{x^{2}-y^{2}}$ orbital and filled $\mathrm{O} 2 p_{x}$ and $2 p_{y}$ orbitals, is the three-band Hubbard model that considers electron hopping $(\mathrm{t})$, the on-site Coulomb interaction $\left(\mathrm{U}_{d}\right)$, the energy difference between oxygen and copper orbitals $\left(\Delta_{p d}\right)$, and the intersite interaction $\left(\mathrm{V}_{p d}\right)^{47,48}$. Anderson proposed that a single-band Hubbard model containing the hopping integral between the nearest-neighbor sites $(t)$ and the on-site Coulomb repulsion (U) can describe the cuprate parent compound ${ }^{49}$. In the case where $U \gg t$ as in the cuprates, the single-band Hubbard model can be further simplified into a one-band $\mathrm{t}-\mathrm{J}$ model which is a possible minimal model for the cuprates ${ }^{50}$. Since the hopping process may also involve the second $\left(\mathrm{t}^{\prime}\right)$ and third $\left(\mathrm{t}^{\prime \prime}\right)$ nearest neighbors, an extended $t-J$ model, the $t-t^{\prime}-t^{\prime \prime}-J$ model, was also proposed ${ }^{51}$. It remains controversial whether the three-band Hubbard model can be simplified into single-band Hubbard model ${ }^{52-54}$. Also, even the single-band Hubbard model or t-J model cannot be solved exactly and approximations have to be applied in the computations. Theoretically, the single-particle spectrum has been mainly calculated from the single-band Hubbard or t-J model. These calculations provide a big picture that agrees well with our measurements on some aspects like the Mott gap structure in the parent compound and the emergence of in-gap states with doping. In this sense, we think that the overall electronic structure evolution can be described by the single-band Hubbard model. However, no solutions of the Hubbard model can reproduce the coexistence of fairly incoherent doped states with large bandwidth extending almost across the charge-transfer gap with a very dispersive lower Hubbard band, as observed in our present experiments. Therefore, our momentum-resolved electronic structures on the parent compound and lightly doped cuprates covering the entire Mott gap region provide key information to examine on these microscopic theoretical models, 
particularly on those that are applied to electron-doped cuprates $43,55-58$.

Our ARPES results reported here touch several central issues on the electronic structure evolution of doping Mott insulators in cuprates. The first is about the nature of the Mott gap. It was suggested that the Mott gap is an indirect gap in cuprate parent compounds $^{55,59}$. Optical ${ }^{60-62}$ and tunneling ${ }^{11,12}$ measurements detected the Mott gap in the parent compounds but cannot distinguish whether it is a direct or indirect gap. By spanning the full gapped region, our momentum-resolved results on the nearly undoped CCOC326 provide direct evidence that the Mott gap is indeed an indirect gap from the top of the charge-transfer band at $(\pi / 2, \pi / 2)$ to the bottom of the upper Hubbard band, consistent with the results from $(\mathrm{Nd}, \mathrm{Ce})_{2} \mathrm{CuO}_{4}{ }^{26}$. The second issue concerns the chemical potential shift upon doping. It has been under debate whether in the lightly electron-doped cuprates, the chemical potential will be pinned inside the gapped region or it will shift to the bottom of the upper Hubbard band ${ }^{9,44}$. Previous results on electron-doped $(\mathrm{Nd}, \mathrm{Ce})_{2} \mathrm{CuO}_{4}$ did not observe the chemical potential jump in lightly doping the parent compound ${ }^{26,63}$. This may be related to the possibility that the parent $\mathrm{Nd}_{2} \mathrm{CuO}_{4}$ sample is not truly undoped. With CCOC326 being a perfect insulator at zero doping, our present work provides direct evidence that, in the parent cuprate compound, upon a tiny electron doping, the chemical potential will jump to the bottom edge of the upper Hubbard band. The third issue is about the nature of the newly formed ingap states. It has been discussed whether or not the in-gap states are coherent quasiparticle states that are confined in a narrow energy range ${ }^{43,55}$. Our present work indicates that the newly developed ingap states are incoherent that spread over a large energy range inside the gapped region. Along the nodal direction, although the in-gap states form a well-defined waterfall-like band (Fig. 2a), the photoemission spectra (EDCs) are broad that spread over a wide energy range. Along the zone face near the antinodal region, although only a narrow band is formed around $(\pi, 0)$ with electron doping (Fig. 2c), the photoemission spectra are broad (Fig. 3b) and the spectral tails extend to a wide energy range. This incoherence of the in-gap states may be related to the strong correlation effect in the lightly doped cuprates. The fourth issue is about the Mott gap. It has been under debate whether, with carrier doping, the Mott gap will change its size or not $^{55}$. Our present work indicates that the Mott gap magnitude changes little with electron doping (Fig. $3 \mathrm{~d}$ ). But it collapses because of the spectral weight depletion of the charge-transfer band and the filling-up of low-lying states inside the gap (Fig. 3a, b, e).

Our momentum-resolved results on lightly electron-doped CCOC326 over the entire gapped region show that the overall electronic structure evolution can be described by the single-band Hubbard model, as shown in Fig. 5e, f. However, the in-gap states behave not as coherent quasiparticles, but as incoherent states that extend over wide energy distribution. In particular, the in-gap states are anisotropic in momentum space; the electronic evolution near the $(\pi / 2, \pi / 2)$ nodal region is distinct from that near the $(\pi, 0)$ antinodal region. The appearance of waterfall-like in-gap states with a large bandwidth along the nodal direction, its position lying below the Fermi level at lower doping, and the gradual approaching of the band towards the Fermi level with increasing doping, cannot be explained by the existing theories ${ }^{43,55-58}$. Our present results ask for further theoretical efforts to propose new theories to understand the electronic structure evolution in lightly doped cuprates, in addition to discriminate against the existing theoretical models.

\section{Methods}

Sample preparation. High quality $\mathrm{Ca}_{3} \mathrm{Cu}_{2} \mathrm{O}_{4} \mathrm{Cl}_{2}$ single crystals were grown by a self-flux method with two steps ${ }^{37}$. First, polycrystalline $\mathrm{Ca}_{3} \mathrm{Cu}_{2} \mathrm{O}_{4} \mathrm{Cl}_{2}$ samples were prepared by conventional solid-state reaction method using high-purity raw materials $\mathrm{CaO}$ (Alfa, 99.95\% pure), $\mathrm{CuCl}_{2}$ (Alfa, 99.995\% pure), and $\mathrm{CuO}$ (Alfa, $99.995 \%$ pure). The powder mixture of $\mathrm{CaO}, \mathrm{CuCl}_{2}$, and $\mathrm{CuO}$ with a molar ratio of $3: 1: 1$ was ground together and heated at $800^{\circ} \mathrm{C}$ for $20 \mathrm{~h}$ with several intermediate grindings in order to obtain single-phase samples. Second, polycrystalline $\mathrm{Ca}_{3} \mathrm{Cu}_{2} \mathrm{O}_{4} \mathrm{Cl}_{2}$ samples were put into an alumina crucible and heated to $1060{ }^{\circ} \mathrm{C}$ with the rate of $180^{\circ} \mathrm{C} / \mathrm{h}$, dwelled at $1060^{\circ} \mathrm{C}$ for $10 \mathrm{~h}$, and cooled to $900{ }^{\circ} \mathrm{C}$ with the rate of $30^{\circ} \mathrm{C} / \mathrm{h}$. The furnace was shut off at $900^{\circ} \mathrm{C}$ to let the samples cool rapidly in order to avoid generating $\mathrm{Ca}_{2} \mathrm{CuO}_{2} \mathrm{Cl}_{2}$ phase.

High-resolution ARPES measurements. High-resolution angle-resolved photoemission measurements were carried out on our lab-based system ${ }^{64,65}$ that is equipped with a Scienta Omicron DA30L electron analyzer. A Scienta Omicron helium discharge lamp with photon energies of $21.2 \mathrm{eV}$ and $40.8 \mathrm{eV}$ was used as the light source. The energy resolution was set at $10-20 \mathrm{meV}$ for Fermi surface mapping and band structure measurements. The angular resolution was $\sim 0.3^{\circ}$. Samples were cleaved at $80 \mathrm{~K}$ and measured at $55 \mathrm{~K}$ to avoid sample charging (see Supplementary Fig. 1). A SAES dispenser is used to evaporate rubidium (Rb) in situ onto the $\mathrm{Ca}_{3} \mathrm{Cu}_{2} \mathrm{O}_{4} \mathrm{Cl}_{2}$ surface step by step. ARPES measurements were carried out after each $\mathrm{Rb}$ deposition and such a process is repeated many times. All measurements were carried out in ultrahigh vacuum with a base pressure better than $5 \times 10^{-11}$ Torr.

\section{Data availability}

The data that support the findings of this study are available from the corresponding author upon reasonable request.

Received: 6 May 2020; Accepted: 29 January 2021; Published online: 01 March 2021

\section{References}

1. Mattheiss, L. F. Electronic band properties and superconductivity in $\mathrm{La}_{2-y}$ $\mathrm{X}_{y} \mathrm{CuO}_{4}$. Phys. Rev. Lett. 58, 1028-1030 (1987).

2. Fink, J., Nucker, N., Romberg, H. A. \& Fuggle, J. C. Electronic structure studies of high- $\mathrm{T}_{c}$ superconductors by high-energy spectroscopies. IBM J. Res. Dev. 33, 372-381 (1989).

3. Zaanen, J., Sawatzky, G. A. \& Allen, J. W. Band gaps and electronic structure of transition-metal compounds. Phys. Rev. Lett. 55, 418-421 (1985).

4. Lee, P. A., Nagaosa, N. \& Wen, X.-G. Doping a Mott insulator: physics of high-temperature superconductivity. Rev. Mod. Phys. 78, 17-85 (2006).

5. Armitage, N. P., Fournier, P. \& Greene, R. L. Progress and perspectives on electron-doped cuprates. Rev. Mod. Phys. 82, 2421-2487 (2010).

6. Dagotto, E. Correlated electrons in high-temperature superconductors. Rev. Mod. Phys. 66, 763-840 (1994).

7. Matsumoto, H., Sasaki, M. \& Tachiki, M. A new narrow band of highly correlated electrons in oxide superconductors. Solid State Commun. 71 829-833 (1989)

8. Allen, J. W. et al. Resonant photoemission study of $\mathrm{Nd}_{2-x} \mathrm{Ce}_{x} \mathrm{CuO}_{4-y}$ : nature of electronic states near the Fermi level. Phys. Rev. Lett. 64, 595-598 (1990).

9. Meinders, M. B., Eskes, H. \& Sawatzky, G. A. Spectral-weight transfer: breakdown of low-energy-scale sum rules in correlated systems. Phys. Rev. B 48, 3916-3926 (1993).

10. van Veenendaal, M. A., Sawatzky, G. A. \& Groen, W. A. Electronic structure of $\mathrm{Bi}_{2} \mathrm{Sr}_{2} \mathrm{Ca}_{1-x} \mathrm{Y}_{x} \mathrm{Cu}_{2} \mathrm{O}_{8+\delta}$ : $\mathrm{Cu} 2 \mathrm{p}$ X-ray-photoelectron spectra and occupied and unoccupied low-energy states. Phys. Rev. B 49, 1407-1416 (1994).

11. Ye, C. et al. Visualizing the atomic-scale electronic structure of the $\mathrm{Ca}_{2} \mathrm{CuO}_{2} \mathrm{Cl}_{2}$ Mott insulator. Nat. Commun. 4, 1365 (2013).

12. Ruan, W. et al. Relationship between the parent charge transfer gap and maximum transition temperature in cuprates. Sci. Bull. 61, 1826-1832 (2016).

13. Kohsaka, Y. et al. Visualization of the emergence of the pseudogap state and the evolution to superconductivity in a lightly hole-doped Mott insulator. Nat. Phys. 8, 534-538 (2012).

14. Cai, P. et al. Visualizing the evolution from the Mott insulator to a chargeordered insulator in lightly doped cuprates. Nat. Phys. 12, 1047-1051 (2016).

15. Zhong, Y. et al. Direct visualization of ambipolar Mott transition in cuprate $\mathrm{CuO}_{2}$ planes. Phys. Rev. Lett. 125, 077002 (2020).

16. Damascelli, A., Hussain, Z. \& Shen, Z. X. Angle-resolved photoemission studies of the cuprate superconductors. Rev. Mod. Phys. 75, 473-541 (2005).

17. Wells, B. O. et al. E versus $\mathrm{k}$ relations and many body effects in the model insulating copper oxide $\mathrm{Sr}_{2} \mathrm{CuO}_{2} \mathrm{Cl}_{2}$. Phys. Rev. Lett. 74, 964-967 (1995). 
18. Ronning, F. et al. Photoemission evidence for a remnant Fermi surface and a d-wave-like dispersion in insulating $\mathrm{Ca}_{2} \mathrm{CuO}_{2} \mathrm{Cl}_{2}$. Science 282, 2067-2072 (1998).

19. Ronning, F. et al. Universality of the electronic structure from a half-filled $\mathrm{CuO}_{2}$ plane. Phys. Rev. B 67, 035113 (2003).

20. Rösch, O. et al. Polaronic behavior of undoped high- $\mathrm{T}_{c}$ cuprate superconductors from angle-resolved photoemission spectra. Phys. Rev. Lett. 95, 227002 (2005).

21. $\mathrm{Hu}, \mathrm{C}$. et al. Evidence for multiple underlying Fermi surface and isotropic energy gap in the cuprate parent compound $\mathrm{Ca}_{2} \mathrm{CuO}_{2} \mathrm{Cl}_{2}$. Chin. Phys. Lett. 35, 067403 (2018).

22. Yoshida, T. et al. Metallic behavior of lightly doped $\mathrm{La}_{2-x} \mathrm{Sr}_{x} \mathrm{CuO}_{4}$ with a Fermi surface forming an arc. Phys. Rev. Lett. 91, 027001 (2003).

23. Shen, K. M. et al. Missing quasiparticles and the chemical potential puzzle in the doping evolution of the cuprate superconductors. Phys. Rev. Lett. 93, 267002 (2004).

24. Shen, K. M. et al. Nodal quasiparticles and antinodal charge ordering in $\mathrm{Ca}_{2-x}$ $\mathrm{Na}_{x} \mathrm{CuO}_{2} \mathrm{Cl}_{2}$. Science 307, 901-904 (2005).

25. Peng, Y. Y. et al. Disappearance of nodal gap across the insulatorsuperconductor transition in a copper-oxide superconductor. Nat. Commun. 4, 2459 (2013)

26. Armitage, N. P. et al. Doping dependence of an n-type cuprate superconductor investigated by angle-resolved photoemission spectroscopy. Phys. Rev. Lett. 88, 257001 (2002).

27. Hossain, M. A. et al. In situ doping control of the surface of high-temperature superconductors. Nat. Phys. 4, 527-531 (2008).

28. Fournier, D. et al. Loss of nodal quasiparticle integrity in underdoped $\mathrm{YBa}_{2} \mathrm{Cu}_{3} \mathrm{O}_{6+x}$. Nat. Phys. 6, 905-911 (2010).

29. Zhang, Y. X. et al. In situ carrier tuning in high temperature superconductor $\mathrm{Bi}_{2} \mathrm{Sr}_{2} \mathrm{CaCu}_{2} \mathrm{O}_{8+\delta}$ by potassium deposition. Sci. Bull. 61, 1037-1043 (2016).

30. Comin, R. et al. $\mathrm{Na}_{2} \mathrm{IrO}_{3}$ as a novel relativistic Mott insulator with a $340-\mathrm{meV}$ gap. Phys. Rev. Lett. 109, 266406 (2012).

31. Kim, Y. K. et al. Fermi arcs in a doped pseudospin-1/2 Heisenberg antiferromagnet. Science 345, 187-190 (2014).

32. Kim, Y. K. et al. Observation of a d-wave gap in electron-doped $\mathrm{Sr}_{2} \mathrm{IrO}_{4}$. Nat. Phys. 12, 37-41 (2016).

33. Miyata, Y. et al. High-temperature superconductivity in potassium-coated multilayer FeSe thin films. Nat. Mater. 14, 775-779 (2015).

34. Wen, C. H. P. et al. Anomalous correlation effects and unique phase diagram of electron-doped FeSe revealed by photoemission spectroscopy. Nat. Commun. 7, 10840 (2016).

35. Kyung, W. S. et al. Enhanced superconductivity in surface-electrondoped iron pnictide $\mathrm{Ba}\left(\mathrm{Fe}_{1.94} \mathrm{Co}_{0.06}\right)_{2} \mathrm{As}_{2}$. Nat. Mater. 15, 1233-1236 (2016).

36. Sowa, T., Hiratani, M. \& Miyauchi, K. A new chlorooxocuprate, $\mathrm{Ca}_{3} \mathrm{Cu}_{2} \mathrm{O}_{4} \mathrm{Cl}_{2}$, with an oxygen defect intergrowth. J. Solid State Chem. 84, 178-181 (1990).

37. Zhao, J. F. et al. Orbital selection of the double $\left[\mathrm{CuO}_{2}\right]$ layer compound $\mathrm{Ca}_{3} \mathrm{Cu}_{2} \mathrm{O}_{4} \mathrm{Cl}_{2}$. Sci. China Phys. Mech. Astron. 62, 107421 (2019).

38. Song, D. et al. Electron number-based phase diagram of $\mathrm{Pr}_{1-x} \mathrm{LaCe}_{x} \mathrm{CuO}_{4-\delta}$ and possible absence of disparity between electron- and hole-doped cuprate phase diagrams. Phys. Rev. Lett. 118, 137001 (2017).

39. Jin, C. Q. et al. Superconductivity at $80 \mathrm{~K}$ in $(\mathrm{Sr}, \mathrm{Ca})_{3} \mathrm{Cu}_{2} \mathrm{O}_{4+\delta} \mathrm{Cl}_{2-y}$ induced by apical oxygen doping. Nature 375, 301-303 (1995).

40. Zenitani, Y. et al. Superconductivity in $(\mathrm{Ca}, \mathrm{Na})_{2} \mathrm{CaCu}_{2} \mathrm{O}_{4} \mathrm{Cl}_{2}$ - the new simplest double-layer cuprate with apical chlorine. Phys. C 248, 167-170 (1995).

41. Segawa, K. \& Ando, Y. Doping n-type carriers by La substitution for $\mathrm{Ba}$ in the $\mathrm{YBa}_{2} \mathrm{Cu}_{3} \mathrm{O}_{y}$ system. Phys. Rev. B 74, 100508(R) (2006).

42. Ikeda, $M$. et al. Chemical potential jump between the hole-doped and electron-doped sides of ambipolar high- $\mathrm{T}_{c}$ cuprate superconductors. Phys. Rev. B 82, 020503(R) (2010).

43. Kusunose, H. \& Rice, T. M. Single-particle spectrum in the electron-doped cuprates. Phys. Rev. Lett. 91, 186407 (2003).

44. Georges, A., Kotliar, G., Krauth, W. \& Rozenberg, M. J. Dynamical mean-field theory of strongly correlated fermion systems and the limit of infinite dimensions. Rev. Mod. Phys. 68, 13-125 (1996).

45. Imada, M., Fujimori, A. \& Tokura, Y. Metal-insulator transitions. Rev. Mod. Phys. 70, 1039-1263 (1998).

46. Phillips, P. Colloquium: Identifying the propagating charge modes in doped Mott insulators. Rev. Mod. Phys. 82, 1719-1742 (2010).

47. Emery, V. J. Theory of high- $T_{c}$ superconductivity in oxides. Phys. Rev. Lett. 58, 2794 (1987).

48. Varma, C. M., Schmitt-Rink, S. \& Abrahams, E. Charge transfer excitations and superconductivity in ionic metals. Solid State Commun. 62, 681-685 (1987).
49. Anderson, P. W. The resonating valence bond state in $\mathrm{La}_{2} \mathrm{CuO}_{4}$ and superconductivity. Science 235, 1196-1198 (1987).

50. Zhang, F. C. \& Rice, T. M. Effective Hamiltonian for the superconducting $\mathrm{Cu}$ oxides. Phys. Rev. B 37, 3759(R) (1988).

51. Tohyama, T. \& Maekawa, S. Angle-resolved photoemission in high $\mathrm{T}_{c}$ cuprates from theoretical viewpoints. Supercond. Sci. Technol. 13, R17 (2000).

52. Emery, V. J. \& Reiter, G. Quasiparticles in the copper-oxygen planes of high$\mathrm{T}_{c}$ superconductors: an exact solution for a ferromagnetic background. Phys. Rev. B 38, 11938(R) (1988).

53. Eskes, H. \& Sawatzky, G. A. Single-, triple-, or multiple-band Hubbard models. Phys. Rev. B 44, 9656 (1991).

54. Varma, C. M. Non-Fermi-liquid states and pairing instability of a general model of copper oxide metals. Phys. Rev. B 55, 14554 (1997).

55. Kusko, C., Markiewicz, R. S., Lindroos, M. \& Bansil, A. Fermi surface evolution and collapse of the Mott pseudogap in $\mathrm{Nd}_{2-x} \mathrm{Ce}_{x} \mathrm{CuO}_{4-\delta}$. Phys. Rev. B 66, 140513(R) (2002).

56. Tohyama, T. Asymmetry of the electronic states in hole- and electron-doped cuprates: exact diagonalization study of the $\mathrm{t}-\mathrm{t}^{\prime}-\mathrm{t}^{\prime \prime}-\mathrm{J}$ model. Phys. Rev. B 70, 174517 (2004).

57. Xiang, T. et al. Intrinsic electron and hole bands in electron-doped cuprate superconductors. Phys. Rev. B 79, 014524 (2009).

58. Weber, C., Haule, K. \& Kotliar, G. Strength of correlations in electron- and hole-doped cuprates. Nat. Phys. 6, 574-578 (2010).

59. Tohyama, T. \& Maekawa, S. Electronic states in the antiferromagnetic phase of electron-doped high- $\mathrm{T}_{c}$ cuprates. Phys. Rev. B 64, 212505 (2001).

60. Uchida, $\mathrm{S}$. et al. Optical spectra of $\mathrm{La}_{2-x} \mathrm{Sr}_{x} \mathrm{CuO}_{4}$ : effect of carrier doping on the electronic structure of the $\mathrm{CuO}_{2}$ plane. Phys. Rev. B 43, 7942 (1991).

61. Arima, T., Tokura, Y. \& Uchida, S. Optical spectra of $\operatorname{Pr}_{2-x} \mathrm{Ce}_{x} \mathrm{CuO}_{4-\delta}$ crystals: evolution of in-gap states with electron doping. Phys. Rev. B 48, 6597 (1993).

62. Waku, $\mathrm{K}$. et al. Charge dynamics of $\mathrm{Ca}_{2-x} \mathrm{Na}_{x} \mathrm{CuO}_{2} \mathrm{Cl}_{2}$ as a correlated electron system with the ideal tetragonal lattice. Phys. Rev. B 70, 134501 (2004).

63. Harima, $\mathrm{N}$. et al. Chemical potential shift in $\mathrm{Nd}_{2-x} \mathrm{Ce}_{x} \mathrm{CuO}_{4}$ : contrasting behavior between the electron- and hole-doped cuprates. Phys. Rev. B 64, 220507(R) (2001).

64. Liu, G. D. et al. Development of a vacuum ultraviolet laser-based angleresolved photoemission system with a superhigh energy resolution better than $1 \mathrm{meV}$. Rev. Sci. Instrum. 79, 023105 (2008).

65. Zhou, X. J. et al. New developments in laser-based photoemission spectroscopy and its scientific applications: a key issues review. Rep. Prog. Phys. 81, 062101 (2018).

\section{Acknowledgements}

We thank the financial support from the National Natural Science Foundation of China (Grant no. 11888101), the National Key Research and Development Program of China (Grant no. 2016YFA0300300 and 2017YFA0302900), the Strategic Priority Research Program (B) of the Chinese Academy of Sciences (Grant no. XDB25000000), the Youth Innovation Promotion Association of CAS (Grant no. 2017013), and the Research Program of Beijing Academy of Quantum Information Sciences (Grant no. Y18G06).

\section{Author contributions}

X.J.Z. and C.H. proposed and designed the research. C.H. carried out the ARPES experiments with Q.G. and H.T.Y. J.F.Z. and C.Q.J. grew the $\mathrm{Ca}_{3} \mathrm{Cu}_{2} \mathrm{O}_{4} \mathrm{Cl}_{2}$ single crystals C.H., Q.G., H.T.Y., H.T.R., J.W.H., J.L., Y.Q.C., C.L., H.C., L.Z., G.D.L., Z.Y.X., and X.J.Z contributed to the development and maintenance of the Laser-ARPES system. T.X. contributed to theoretical analysis. C.H. and X.J.Z. wrote the paper. All authors participated in discussions and comments on the paper.

\section{Competing interests}

The authors declare no competing interests.

\section{Additional information}

Supplementary information The online version contains supplementary material available at https://doi.org/10.1038/s41467-021-21605-6.

Correspondence and requests for materials should be addressed to C.J. or X.J.Z.

Peer review information Nature Communications thanks Rui-Hua He and the other, anonymous, reviewer(s) for their contribution to the peer review of this work.

Reprints and permission information is available at http://www.nature.com/reprints

Publisher's note Springer Nature remains neutral with regard to jurisdictional claims in published maps and institutional affiliations. 
(c) (i) Open Access This article is licensed under a Creative Commons Attribution 4.0 International License, which permits use, sharing, adaptation, distribution and reproduction in any medium or format, as long as you give appropriate credit to the original author(s) and the source, provide a link to the Creative Commons license, and indicate if changes were made. The images or other third party material in this article are included in the article's Creative Commons license, unless indicated otherwise in a credit line to the material. If material is not included in the article's Creative Commons license and your intended use is not permitted by statutory regulation or exceeds the permitted use, you will need to obtain permission directly from the copyright holder. To view a copy of this license, visit http://creativecommons.org/ licenses/by/4.0/.

(C) The Author(s) 2021 